\title{
On the Analysis and Application of Context Function in English Linguistics
}

\author{
Linlin Tong \\ Tianshi College Tianjin China 301700 College English Excellent course JP20140002
}

Keywords: Context analysis; Context function classification; linguistics

\begin{abstract}
Context feature exists in every language and context is an important reference for the semantic interpretation in daily language communication, in-depth understanding of the functional classification of context, the rational use of contextual assumptions, helps to enhance the understanding of language and communication skills. As a language learner learning guidance and management teachers how to use the functional differences between the different context to interpret the actual semantics, enable it to have a flexible and do not break the law, to let the students experience language the true meaning and charm, it is need to study and continue to explore the topic. The to make detailed analysis on the function of different context, to investigate the classification, looking for language in the specific context of the use of rules, seeking an explanation of semantic optimization approach, for the students' language learning, use of lay a solid foundation.
\end{abstract}

\section{Introduction}

Traditional blunt Semantic Contrast and grammatical phrases to recite, through mechanical repetition, formed a kind of mode of thinking in Chinese English learning mode, this lack of flexible teaching methods and context creation of rigid way, the actual language of the students communication skills improve the disadvantages outweigh the advantages. Therefore, we need to gradually establish a new model of language learning, that is, the English language is used to express the user's ideas, highlighting the user's purpose, plans, and other communication functions of a tool. Only in depth understanding of the meaning and classification of context, is the key to develop a good sense of language.

\section{Context Classification}

Context is the context of language expression, which includes two factors: linguistic and nonlinguistic factors. Context is an important reference for understanding the meaning and the use of language in daily language communication, and it is also the subjective factors that affect the performance of the language. Usually the influence factors of the context include: the context of cohesion, time, place, object, context, the context of discourse, etc. [1] Specifically speaking, context can be divided into the following four categories:

Verbal Context. Verbal context, that is, the most basic through the English word, word, sentence, paragraph of the meaning of the sign or the meaning, combined with the context to help understand the expression of the specific meaning and direction of the expression. For example:" Dad, where is my jacket?" "Oh, your mom just come to clean up the room!" In this brief conversation, the son asks his father where he is. The father did not tell his son where the jacket was, but the boy had already known the answer from his answer. This is the context of the reasoning and verbal context of the language reference, the son can be speculated that the jacket has been taken away.

Situational Context. Situational context refers to the nonlinguistic factors that are associated with the location or context of the conversation. Under the influence of this kind of factor, even if it is the same sentence, under the different scene mapping, also can have the different meaning. As:" He got to the bank", If do not have a specific context as a reference, who also don't know the meaning of this sentence, whether it is "he went to the bank", or "he went to the river bank". [2] 
This is the context of the impact of the context of the semantic role and reference, the expression of the semantic meaning of the speaker's emotions will be different with the situation, in different ways.

Cultural Context. Cultural context can be said that many of the context of the category is more complex, short time is difficult to use and master the use of context categories. It will be based on the cultural background of the language system, and produce a lot of symbols, ceremonies and occasions, a huge difference. Under the influence of the cultural background, even if the same word, word, sentence, including the thoughts or feelings or reflect the role of the exchange is also different. Especially in the huge differences between Chinese and Western cultural development, cultural differences are very obvious, and the characterization of the cultural context is also very prominent. Just like "white yarn the" white yarn, in China, mostly in the funeral ceremony will be used in the cloth, to express the grief and loss of their loved ones. In the west, the white yarn is a symbol of purity, beauty, freedom. So in the western white yarn instead mostly used to make wedding dress, indicates that the husband and wife between the pure love and good wishes for the future. Such as Phoenix "phoenix", in the East represents the beauty and elegance, while in the West it is the meaning of "resurrection and rebirth".

Secondary Language. Assistants to the vice language, mainly referring to is accompanied by changes in the speaker's utterance displayed through intonation, stress, tone, speed, eye contact, gestures, facial expressions and body movements etc.. The form is more abundant, but it is the auxiliary expression of the speaker's mood and attitude, and related to the context of the. This often occurs in the life of the English language, this is not much to say.

\section{Analysis of the Application of the Function of English Context}

Limiting Function. Hedges can be broadly understood as the description of things is not clear enough, prone to ambiguity, ambiguity of the extent of the word limit. Fuzzy language in the analysis of the relevant experts and research, roughly divided into two categories, ease of shape and change of hedges.

Relaxation type restriction is to express the views and attitudes of the speaker on the subject by using the degree of subjective guess or by using the third party's point of view as the basis. With a euphemism, to a certain extent, can ease the tension of the atmosphere, with the implicit way to maintain the peace of the two sides, maintaining image, break the deadlock and so on. For example," According to the network, the population will increase next year." It is not difficult to see that is the use of hedges, to ease the speaker's degree of certainty, so as not to attract others refute or dissatisfaction. [3]

The change type fuzzy language often appears in the news report and the newspaper is one kind of universal language phenomenon. Readers from the psychological and social point of view of this kind of fuzzy language understanding and analysis, the reader's knowledge structure and social cognition, and even the value of his understanding will play a decisive role. So, every reader can interpret meaning not the same, this is a relevance adaptation process, dynamic process of readers in the semantic context adaptation reasoning continue to make the presumption of relevance, in order to find the best answer.

Contrast Supplementary Sentence Meaning. In the expression of all languages, the presence of the indicator is very important. However, in the context of context, the indicator is extremely strong, once out of the relevant context, the role of the indicator cannot be determined. As in the same article, the relative person pronoun is very much, and the same pronoun "he" "she" "it" refers to the object must rely on the context meaning.

In addition to the functions of the control, the English language context also has the complement function to the semantics. This function can be realized through the above four kinds of contexts. Supplement can be divided into two kinds, one kind is the "implied" supplement. For example" where is my pen?"' Oh, I don't know, but I just saw he came out from your house", the answer of the question on the one hand, to highlight their "don't know", on the other hand made suggestive added, "he just from the room out. Although there is no direct answer to his questions, but questions 
from the respondents through the context that a suggestive answer. For example, in a sentence containing cultural context: I meet my Waterloo in my business. Obviously with various meanings can explain with "Waterloo" which contains allusions, historical and cultural background can understand the semantics of the sentence. It can be seen that the use of contextual function is not a simple type, often in the practical application of a variety of different context functions of the comprehensive use of cross. Thus these types have a more profound understanding of the two sides, when talking to you. There is also a category of "omission" supplement, most of the daily oral composition of the sentence to streamline, so that the exchange is more convenient.

The Application of Communicative Context in English. People in the study of language, often pay attention to the interpretation of the meaning of the sentence, while ignoring the language is the most important function is the exchange. Language is due to the needs of the exchange between the two sides, so the context of the application, the natural need to return to the actual use of spoken language. [4] And the use of oral English in our country, the main business negotiation and the two categories of the Multi-National Corporation.

Business English is the most typical kind of communication. In this kind of language communication, because it involves a lot of slang and idioms, elliptical sentences and symbolic sentences, the function of cultural context is very important. It can be said that English level reached a certain level, such as professional English language to students, improve the English level has not only limited to understand the meaning of and the semantic interpretation of surface, depending on the outside of the language culture background knowledge level of understanding, which also in a certain extent determines the success or failure of business communication.

If in this formal occasion, the lack of understanding of the cultural background of the language, the two sides cannot talk even the most basic consensus cannot be reached. For example, when "must" is used in Chinese, it means "must", but more in a foreign language is a kind of suggestion or test:

A: We must get more talk to do the decision.

B: Thank you!

This is typical of the cultural context of the lack of a joke. In fact, he did not understand the meaning of the sentence is wrong, but it ignores the cultural background caused by language habits. These are textbooks cannot be expressed, the need to practice the experience of the application of the context function. Or, the most typical business activities in regards to the western people do not like to use the "hard", must be tired." Sentences, and more like to use "about your journey How I hope you will have a nice day here!" is the same as the culture of the.

\section{Classroom Reform Based on Context Function}

These two points, the function and application of the context in English linguistics have been described. However, the current college education reform has yet to put on the agenda. The important role of context cannot be effectively delivered to the students, which makes the education and training of students, the lack of practical ability to use language, it is difficult to meet the needs of the future language environment.[5] And the in-depth analysis of the linguistic context functions in many places the use of urgently felt the change form of class, teaching form change, change the students' thinking is in the arrow on the string, from the inside, from the teachers themselves and make the first move, change is the most important.

In the process of classroom teaching, teachers mainly through the exchange of language to complete the task. Therefore, teachers in the classroom language use, we must consider the context of the part, and in this way to create the most close to the actual English learning atmosphere for students. Teachers can use the language communication in the classroom to join a variety of context, so that students learn to jump out of the word, the sentence itself, a more profound understanding of the meaning of. Thus from the rigid old translation learning, so that students develop a good habit of thinking in English. Encourage students to learn more about the history of English culture, so as to achieve a higher level of achievement in language learning.

Language learning is never a simple word correspondence, but has more rich and profound 
cultural connotation and connotation. As an important tool for communication and exchange of daily life, the ultimate goal of language learning is the application and all language learning can not be divorced from context, pay attention to the context in the language learning reference value, in order to truly enhance students' English language ability and language literacy.

\section{References}

[1] Zhang Lihui explores the limit function of context and its application in English teaching [J]$2015(7)$

[2] Pragmatic functions of [2] Jia Manli hedges in English classroom teaching [J]- Journal of Nanyang Normal University 2009 (8)

[3] Zhang Qingdong systemic functional linguistics context theory in College English teaching [D] Jilin University 2007

[4] The analysis and application of [J]-'s new curriculum (education Academic) 2010 (3): [4]

[5] $\mathrm{Yu}$ Bo's analysis and application of contextual function in English Linguistics [D] Liaoning Normal University 2004 